\title{
Ueber stereoskopische Messung ${ }^{1}$ ).
}

\author{
Von
}

\author{
Dr. L. Heine,
}

Privatdocenten und I. Assistenten der Universitäts-Augenklinik in Breslau.

Mit Taf. IX u. X, Fig. $5-12$ und 4 Figuren im Text.

Die im Folgenden mitzutheilenden Untersuchungen über stereoskopische Messung schliessen sich eng an meine früheren Mittheilungen über "Orthosterenskopie", „Orthoskopie" und "Sehschärfe und Tiefenwahrnehmung" an (siehe dieses Archiv, Bd. LIII, S. 306, Bd. LI, S. 563 und Bd. LI, S. 146).

Um mich nicht beständig zu wiederholen, setze ich die Kenntniss der Ergebnisse obiger Mittheilungen der Hauptsache nach voraus. In diesen früheren Arbeiten habe ich mich des Genaueren über die Unterschiede der monocular bestimmbaren Sehschärfe gegenüber der binocularen Function der Tiefenwahrnehmung verbreitet.

1) Anmerkung während der Correctur: In Band LIV dieses Archivs, Heft 3 , ist ein "Weiterer Beitrag zur Kemntniss der binocularen Tiefenwahrnehmung" von Elschnig erschienen, auf den ich später genauer eingehen werde. Für heute möchte ich nur bemerken, dass ich weit entfernt bin, den Satz, der meine Ansicht kurz zusammenfassen soll (S. 413), anzuerkennen: „die Entfernung, in der wir im Stereoskop das Ganzbild sehen, ist abhängig vom Grade der Convergenz." Ich hoffe zeigen zu können, woher dieses Missverständniss kommen konnte.

Ferner sagt Elschnig (S. 423): „Durch die Verwendung von Prismen wird die Wirkung der Querdisparation ungebührlich in den Vordergrund geschoben, die zeichnerische Unregelmässigkeit dagegen geradezu ausgeschaltet..." das ist's aber gerade, was ich beabsichtigte: Isolirt die Bedeutung der Querdisparation zu untersuchen. Ist es doch anerkannter Grundsatz der physiologischen Methodik, die Versuchsbedingungen denkbar zu vereinfachen, nicht zu compliciren. Die Bedeutnng der Längsansdehnung für die Stereoskopie hat auch mich seit längerer Zeit beschäftigt, doch sind das andere Dinge, die auch v. Helmholtz noch nicht getrennt hat. Meine Untersuchungen darüber werde ich bald mittheilen können.

Ich glaube in der That, dass dann die wesentlichsten Differenzpunkte ihre Erledigung oder doch Erklärung finden dürften. 
Wir haben gesehen, dass für erstere, so weit wir sie mit der Doppelobjectmethode bestimmen (Snellen'sche Buchstaben und Aehnliches), durch die der Helmboltz'schen Winkelminute entsprechenden Werthe eine Grenze gesetzt ist; wir hatten ferner gesehen, dass diese Grenze für die Tiefenwahrnehmung nicht existirt, dass wir hierfür ein über zehnfach feineres Unterscheidungsvermögen haben, dass wir also für die Wahrnehmung der dritten Dimension in der Zapfenbreite oder der dazu gehörigen Winkelminute keine Grenze haben.

Waren somit die physiologischen Bedingungen für die Wahrnehmung der dritten Dimension präcisirt, so trat die Frage an uns heran, wann sehen wir die dritte Dimension richtig, d. h. unter welchen Bedingungen bilden wir uns ein richtiges Urtheil über die Tiefenverhältnisse (Körperlichkeit, Plastik) eines dreidimensionalen Gegenstandes? Wir können ein verticales Prisma, welches uns eine Kante zukehrt, körperlich als solches erkennen, wir haben aber noch nicht ein richtiges Urtheil über seine Plastik, wir können z. B. ein gleichseitiges Prisma für rechtwinklig halten, unterschätzen also seine Tiefenwerthe, und umgekehrt. Dies führte uns zur Formulirung der Sätze über die Abhängigkeit der relativen Entfernungsschätzungen von der Vorstellung absoluter Entfernung. Wir fanden hier einen weitgehenden Einfluss des empirischen Momentes in der Taxirung der dritten Dimension. Einen Seitenweg an dieser Stelle der Untersuchungen schlug Weinhold auf meine Veranlassung ein (dieses Archiv, Bd. LIV, S. 201), indem er zu bestimmen suchte, welches das primäre sei; die Unterscheidung wenig differenter Eindrücke beider Augen oder die Verschmelzung derselben zu einer höheren Einheit, einer Tiefenvorstellung. Er stellte durch geeignete Prismencombinationen die horizontalen Meridiane beider Augen über einander, so dass die verticalen Meridiane in einer Verticalen über einander zu stehen kamen, und schaltete so das Zustandekommen einer Tiefenwahrnehmung aus. Längsdisparate Doppelbilder horizontaler Contouren erkannte er jetzt als solche, wenn die Längsdisparation wenig über eine Winkelminute stieg. Er schliesst daraus, dass wir primär veranlagt sind, wenig differente Eindrücke beider Augen zu unterscheiden, d. h. in ihre einzelnen Componenten aufzulösen, und dass, falls eine Tiefenwahrnehmung möglich ist, die Verschmelzung solcher wenig differenter Eindrücke ein secundärer Vorgang, nicht aber das Unterlassen einer Differenzirung ist.

In Fortführung der Untersuchungen über das richtige Körperlichsehen (Orthoskopie) suchte ich experimentell die Bedingungen fest- 
zustellen, unter denen wir zwei stereoskopische Halbbilder zu einem dem Original in seinen Tiefenverhältnissen entsprechenden körperlichen Sammelbilde verschmelzen. Wir kamen zu relativ einfachen Sätzen, unter welchen Bedingungen ein dreidimensionaler Gegenstand photographisch aufgenommen werden muss, und unter welchen Bedingungen die Wiedervereinigung beider stereoskopischer Halbbilder zu erfolgen hat, um das Bild in seiner richtigen Plastik erscheinen zu lassen. Zumal bei diesen letzteren Versuchen zeigte sich wieder ein erheblicher Einfluss subjectiver, individuell wohl verschiedener Momente, die sich vielleicht bisher noch nicht völlig durchschauen liessen, und die auf eine weitgehende Abhängigkeit der Bewerthung der seitlichen Bildverschiedenheiten (der Querdisparationen) von der Vorstellung der absoluten Entfernung hinwiesen, in die wir, mehr oder weniger unbewusst, das Sammelbild verlegen. Wir verglichen das körperliche Sammelbild mit dem körperlichen Gegenstand, indem wir ersteres mit letzterem zur Deckung brachten. So konnten wir noch am ehesten zu einem Urtheil kommen, ob die Tiefenverhältnisse beider übereinstimmten. Aber auch bei dieser Methode ist die Beurtheilung oft recht schwierig und erfordert viel Uebung. Dieses legte den Wunsch nahe, Methoden zu suchen, nach denen wir unter möglichster Ausschaltung solcher subjectiven Momente die Tiefendimensionen stereoskopischer Sammelbilder zahlenmässig messen können.

Theoretisch sehe ich hier drei Wege, von denen die zwei ersten kurz besprochen, der dritte seiner praktischen Bedeutung wegen etwas ausführlicher behandelt werden soll. Wir werden zu reden haben

1. von der stereoskopischen Berechnung,

2. von der stereoskopischen Reconstruction,

3. von der stereoskopischen Messung im engeren Sinne mittels Scala.

\section{Die stereoskopische Berechnung.}

Von drei verticalen Stäben, welche durch ihre Anordnung ein gleichseitiges Prisma nachahmen, sei eine Kante dem Doppelauge oder dem stereophotographischen Aufnahmeapparat symmetrisch zugewendet. Dann sind in einer horizontalen Ebene die Abbildungsverhältnisse die der Fig. 1 entsprechenden, $A$ und $B$ nennen wir die Basisstäbe, $C$ entspricht der Höhenkante. Im linken Halbbild liegt $\gamma$ (das Bild von $C$ ) näher an $\beta$ als an $\alpha$. Im rechten Auge liegt $\gamma^{\prime}$ näher an $\alpha^{\prime}$ als an $\beta^{\prime}$. Legen wir beide Bilder auf einander, so deckt sich $\beta$ mit $\beta^{\prime}$, $\alpha$ mit $\alpha^{\prime}$, wicht zur Deckung gelangen $\gamma$ und $\gamma^{\prime}$. Diese

จ. Graefe"s Arehiv fur Ophthalmologie. LV. 2. 
L. Heine.

Strecke $\gamma \gamma^{\prime}$ entspricht der Querdisparation der verticalen Linie $C$ in Bezug auf die Ebene $A B$, mit anderen Worten: aus der Grösse der Querdisparation $\gamma \gamma^{\prime}=d$ können wir berechnen, wie weit $C$ von der Ebene $A B$ entfernt ist, wenn wir wissen, wie weit die Ebene $A B$ rom stereoskopischen Aufnahmeapparat entfernt ist, wie gross die seitliche Objectivdistanz ist, und endlich wie gross die Brenn-

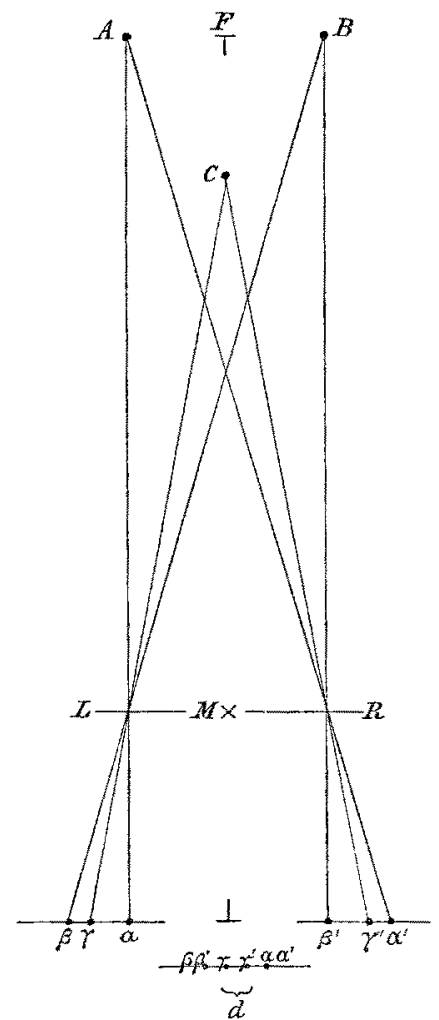

Fig. 1.

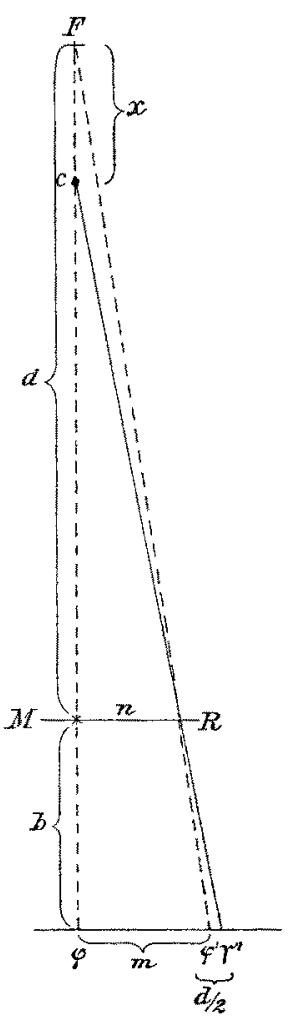

Fig. 2.

weite der benutzten Objective ist. Nennen wir nun die Entfernung des Objectes von den Objectiven $a$, die Balglänge $b$, die halbe $\mathrm{Pu}$ pillendistanz $n$, die Strecke $\varphi \varphi^{\prime} \mathrm{m}, \gamma \gamma^{\prime} \mathrm{d}$, so ist $\varphi^{\prime} \gamma^{\prime}=\frac{d}{2}$.

Nun verhält sich $\frac{a-x}{n}=\frac{a-x+b}{m+\frac{d}{2}}$

und da $m=\frac{n}{a} \cdot(a+b)$ ist, so ist

$$
x=a-\frac{n b}{m+\frac{d}{2}-n}
$$




$$
x=\frac{a^{2} d}{2 b n+a d} .
$$

Die einzige Unbekannte $x$ lässt sich demnach berechnen, wenn wir $\frac{d}{2}$ im Bilde messen können. Praktisch werden wir also im linken stereoskopischen Halbbilde $\beta \gamma$ messen, dann im rechten $\beta^{\prime} \gamma^{\prime}$. Die Differenz ist $=d$, welches als negative Grösse einen Nahewerth, als positive einen Fernwerth haben wiurde.

Nehmen wir schematisch für die Objective eine Brennweite $f .=18 \mathrm{~cm}$ an, so wäre, wenn $a=100 \mathrm{~cm}$ ist, $b=22 \mathrm{~cm}$,

Im ersteren Falle entspräche

$$
" \quad " \quad a=200 " \quad b=20 "
$$

bei einem Werthe $\frac{d}{2}=0,25 \mathrm{~mm} x=35 \mathrm{~mm}$,

$$
" \quad " \quad "=0,1 \mathrm{~mm} x=14 \mathrm{~mm} \text {. }
$$

Im zweiten Falle entspräche

$$
\text { bei einem Werthe } \frac{d}{2}=0,25 \mathrm{~mm} x=150 \mathrm{~mm} \text {, }
$$

$$
" \quad " \quad "=0,1 \mathrm{~mm} x=60 \mathrm{~mm} \text {. }
$$

D. h. bei der Berechnung der Tiefenwerthe eines stereoskopischen Sammelbildes, dessen Halbbilder mit $20 \mathrm{~cm}$-Objectiven bei einer seitlichen Objectivdistanz von $64 \mathrm{~mm}$ aus der Entfernung $100 \mathrm{~cm}$ gewonnen wurden, bedingt ein Messungsfehler von $0,1 \mathrm{~mm}$ einen Rechenfehler von $14 \mathrm{~mm}$ Entfernungsdifferenz, ein Messungsfehler von $1 / 4 \mathrm{~mm}$ einen Rechenfehler von fast $35 \mathrm{~mm}$. War der aufzunehmende Gegenstand nicht 100 , sondern $200 \mathrm{~cm}$ entfernt, so entsprechen denselben Messungsfehlern von 0,1 resp. $0,25 \mathrm{~mm}$ schon Strecken von 60 resp. fast $150 \mathrm{~mm}$.

Für Gegenstände, die aus geringer Entfernung aufgenommen wurden, sind die Fehler wohl kleiner, und wenn wir die Ausmessung der Bilder mittels Mikroskops und Ocularmikrometers machen können, ist noch eine grössere Genauigkeit zu erzielen; immerhin leuchtet ein, dass wir auf diesem Wege kaum zu exacten Resultaten kommen werden. Vollends versagt diese Methode dann, wenn wir keine markanten Punkte oder Linien zur Abmessung benutzen können, sondern wenn es sich um Flächen handelt, die allmählich in einander übergehen.

\section{Die stereoskopische Reconstruction.}

Die zweite Methode, aus stereoskopischen Bildern die Tiefenverhältnisse zahlenmässig festzustellen, möchte ich die der stereoskopischen Reconstruction nennen. Sie ist weit complicirter als 
die Methode der einfachen Berechnung, erfordert einen gewissen Apparat, giebt dafür aber auch sehr genaue Resultate. Wenn wir im Stereoskop anstatt der gewöhnlich gebräuchlichen aufgezogenen Papiercopien Diapositive oder allenfalls die Negativplatten direct zu einem Sammelbilde vereinigen, so können wir, durch Regulirung der Seitendistanz beider Bilder, das Sammelbild an jede Stelle im Raum verlegen. Bei der Durchsichtigkeit der Halbbilder sehen wir ausserdem, was sich sonst etwa an dieser Stelle befindet, z. B. einen Massstab. Nehmen wir nun an, wir hätten einen körperlichen Gegenstand aus $1 \mathrm{~m}$ Entfernung stereophotographisch aufgenommen, so reguliren wir im Stereoskop den seitlichen Abstand beider Negativ- oder Diapositivhalbbilder so, dass das Sammelbild in. $1 \mathrm{~m}$ Entfernung $\mathrm{zu}$ liegen kommt. Der „Rücken" des körperlichen Sammelbildes, d. h. die uns abgewandte Fläche, oben beim Prisma die „Basis"fläche genannt (auch wohl als "Hauptfläche" bezeichnet), wird hier in $1 \mathrm{~m}$ Entfernung etwa mit zwei frontal neben einander stehenden verticalen Stäben in eine Ebene eingestellt. Stenopäische Löcher sorgen dafür, dass Bilder und Stäbe gleichzeitig scharf erscheinen. Eine hinter den Stäben aufgestellte Mattscheibe, am vortheilhaftesten von hinten her hell beleuchtet, sorgt für genügende Helligkeit. Befindet sich nun zwischen den zwei Basisstäben ein Stab, der vom Beobachter aus sagittal verschieblich ist, so kann dieser Stab an die Stelle gebracht werden, wo irgend ein prominenter d.h. uns näher befindlicher Punkt im stereoskopischen Sammelbilde sich befindet. Gleitet dieser mittlere Stab zwischen zwei Schienen, deren eine in Centimeter getheilt ist, so kann man direct die Tiefenverhältnisse des stereoskopischen Sammelbildes ablesen.

Zur praktischen Ausführung braucht man ein linsen- und prismenloses Stereoskop ${ }^{1}$ ) (s. Fig. 3), dessen zwei Pupillen durch stenopäische Löcher von $1 / 2 \mathrm{~mm}$ gebildet sind, deren Distanz durch ein Rechtslinksgewinde regulirbar ist. Auf der Stereoskopschiene sagittal verschieblich ist ein Plattenhalter, in den die Negative bezw. Diapositive eingeschoben werden können und deren Seitendistanz wiederum durch Rechtslinksgewinde regulirbar ist. Eine Höbenverschiebung dieses Platten-

1) Die von mir benutzten Objective (Extra-rapid-lynkeioskop Görz $f=9 \mathrm{~cm}$, Frontlinse abgeschraubt, $f=17,5 \mathrm{~cm}$ ) bedingen eine gewisse Bildverzeichnung im Sinne einer Verkleinerung; dementsprechend steht das Objectiv bei Aufnahmen in natürlicher Grösse, dem Object etwas näher als der Mattscheibe, wenn die Distanz von Object zu Mattscheibe $70 \mathrm{~cm}$ beträgt. Aus dem gleichen Grunde muss im stenopäischen Stereoskop die Negativ- oder Diapositivplatte sich den stenopäischen Löchern etwas näher befinden, als der Balglänge entspricht. 
halters gegen die Blicklinie ist meist entbehrlich. Eine Einbeissvorrichtung und ein Stirnhalter sind öfter erwünscht. Die Platten hatten in meinen Versuchen die Grösse von 5 zu $5 \mathrm{~cm}$. Sie müssen soweit von den stenopäischen Löchern entfernt sein, wie bei der photographischen Aufnahme von den Objectiven, resp. man verschiebt sie im Stereoskop so lange sagittal, bis sie sich mit dem Originalobject der Grösse d. h. der Höhe und Breite nach decken. Das ganze Stereoskop ruht auf einem festen Fuss nach Art der Zehender-Westienschen Lupe.

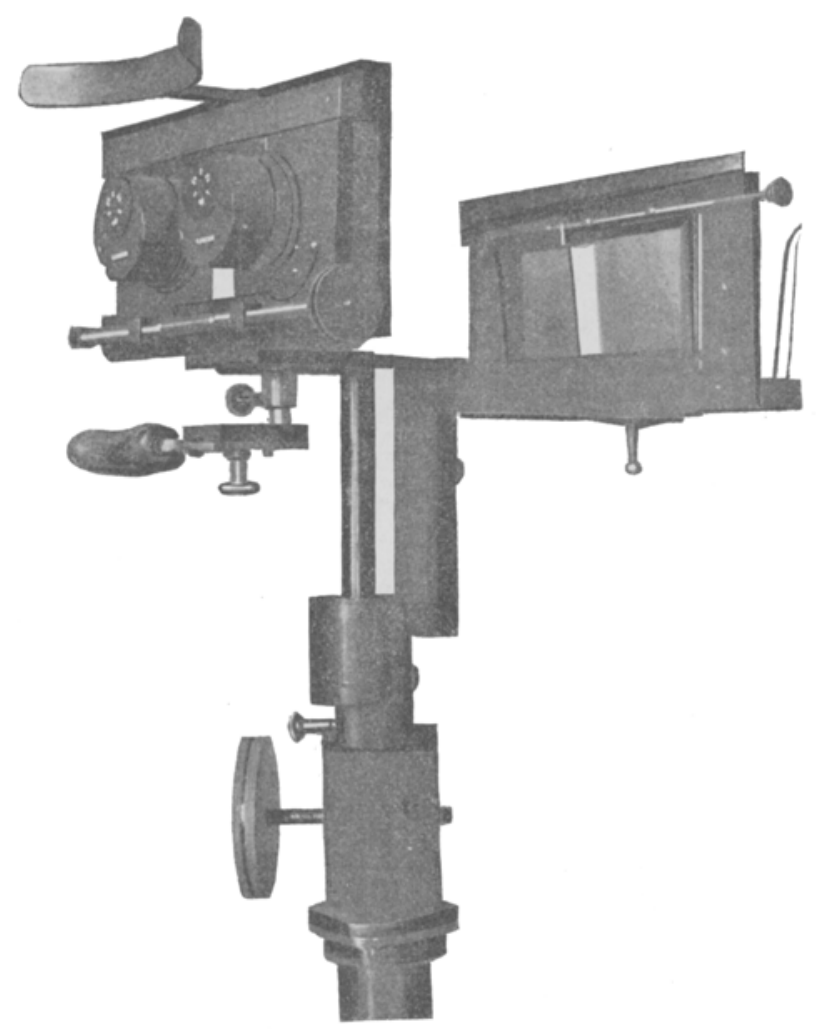

Fig. 3.

Das Verfahren ist allerdings etwas complicirt, giebt dafür aber erstaunlich genaue Resultate. Selbst Beobachter mit keinem hervorragend guten Tiefenwahrnehmungsvermögen können sehr exacte Einstellungen machen. Zunächst ist der „Rücken“ oder die „Basis“ des Sammelbildes mit den beiden Basisstäben in eine Ebene zu bringen. Dieses wird nach genauer Einstellung der Pupillendistanz d. h. der 
seitlichen Distanz der beiden stenopäischen Löcher durch Seitenverschiebung der Halbbilder bewirkt. Man kann auf diese Weise durch Hin- und Herschrauben sich in die richtige Mitte „pendeln", wenn ich mich kurz so ausdrücken darf. Wenn auch die Fehlergrenzen bei bester Tiefenwahrnehmung des Beobachters geringere sind, d. h. wenn der Beobachter mit bester Tiefenwahrnehmung schon bei kleineren Beträgen im Stande ist, eine Tiefenwahrnehmung zu machen, so kann sich doch der mit einer weniger guten Tiefenwahrnehmung ausgestattete mit Hilfe solcher "Pendelungen" die richtige Mitte heraustasten. Dieselben Manipulationen werden dann wiederholt, wenn der mittlere Stab durch sagittale Verschiebungen mit einem näher gelegenen Punkt des Sammelbildes zur Deckung gebracht, oder was vortheilhafter ist, in einer frontalen Ebene jener neben diesen gestellt wird.

Ich habe zu diesem $Z$ wecke stereophotographische Aufnahmen von drei - 1 bis $2 \mathrm{~mm}$ dicken - Stäben gemacht, welche vertical standen, zwei in einer frontalen Ebene, der dritte 2, 4, 6 und mehr $\mathrm{cm}$ vor dieser Ebene. In dem oben geschilderten Stereoskop wurde nun jedes Halbbild durch sagittale und seitliche Verschiebungen an die ihm zukommende Stelle der Gesichtslinie gebracht, so dass der Seitenabstand seiner zwei Basisstäbe mit dem der Basisstäbe selbst gleich gross erschien. Ist dieses für jedes einzelne Auge eingestellt, so muss für die binoculare Betrachtung das Sammelbild der Basisstäbe angenähert in derselben Entfernung stehen, wie die Basisstäbe selbst. Nämlich in derselben Fntfernung, in der die stereophotographische Aufnahme gemacht war. In der Regel wird aber das Sammelbild etwas vor oder hinter der Ebene der beiden Basisstäbe liegen. Die genaue Einstellung des Sammelbildes in die Ebene der beiden Basisstäbe erreichen wir durch die pendelartigen Einstellungen der Halbbilder mit Hilfe des Rechtslinksgewindes. Ist dieses erfolgt, so erscheint also das Sammelbild beider Basisstäbe genau eben so gross (oder breit) wie die Basisstäbe selbst und liegt mit diesen in einer frontalen Ebene. Nun erst schieben wir den dritten Stab zwischen die zwei Basisstäbe ein und verschieben ihn, sagittal pendelnd, so lange hin und her, bis er mit dem Sammelbild des mittleren Stabes in einer frontalen Ebene zu liegen scheint.

So können wir aus stereoskopischen Bildern die Tiefenverhältnisse „reconstruiren". Die Exactheit ist eine fast absolute, d. h. eine Grenze ist hierfür durch die der Zapfenbreite entsprechenden Winkelwerthe ebenso wenig gegeben, wie für die Tiefenwahrnehmung. Eine 
genaue Kenntniss, wie stark die Aufnahmeobjective gewesen sind, ist also für diese Methode dann nicht nöthig, wenn wir wissen, wie hoch oder breit etwa das Object gewesen ist. Es genügt dieses für die Regulirung der Entfernung zwischen Halbbildern und stenopäischen Löchern im Stereoskop.

\section{Die stereoskopische Messung mittels Scala.}

Die dritte Methode bezweckt die stereoskopische Ausmessung aufgeklebter Stereogramme mittels einer auf die Bilder aufzulegenden Messscala. An dieser Stelle berühren sich meine Versuche mit denen Pulfrich's, welche in der optischen Werkstätte von Zeiss unternommen wurden und zur Construction der stereoskopischen Entfernungsmesser führten: Diese Instrumente dienen zur binocularen Ausmessung der Tiefenverhältnisse in der Wirklichkeit, z. B. in einer Gegend. Zwei Fernrohre entwerfen je ein stereoskopisches Halbbild in ihren Brennebenen. In diesen letzteren befinden sich die Halbbilder einer berechneten Scala, welche binocular verschmolzen, den Eindruck einer in die Tiefe verlaufenden Markenreihe machen. Gleichzeitig mit dem Sammelbild der Wirklichkeit (z. B. der Gegend) sehen wir also im Fernrohr eine Markenreihe, welche wie Meilensteine die Entfernungen abzulesen gestattet.

Vorausgesetzt, dass die geringsten mit der Scala abzulesenden Entfernungen in Bezug auf die Objectivbrennweite gleich unendlich zu. sehen sind, sich also etwa in der Brennebene abbilden, kommt man mit einer einzigen - leicht zu berechnenden - in dieser Brennebene aufzustellenden Scala aus.

„Eine Anwendung des Stereoskops, welche sehr nahe liegt und bisher noch nicht ausgeführt ist, wäre die zur Schätzung und Messung von Raumgrössen. Bringt man einen beliebigen Körper und etwa das Drahtmodell eines Cubikfusses, der in Cubikzoll abgetheilt ist, neben einander und dazwischen ein unbelegtes Planglas, so scheint der Cubikfuss den Körper zu durchdringen und es ist nicht schwer, Schätzungen oder Messungen an dem Körper auf diese Weise vorzunehmen.

„Aehnlich muss es nun sein, wenn man durch ein solches cubisches Netz, welches stereoskopisch auf Glas abgebildet ist, in den Raum hinaussieht. Es werden dann die Gegenstände einfach von diesem Netz durchdrungen.

... „Die Verbindung mit dem Telestereoskop wäre für manche Fälle zweckmässig $\left.{ }^{1}\right)^{46}$

1) Mach in Sitzungsber. der d. k. Acad. Math.-nat. Kl. Bd.LIV. Abth. 2. 1866. 
Die Priorität der Idee des stereoskopischen Entfernungsmessers scheint demnach Mach zu gebühren ${ }^{3}$.

In meinen hier darzulegenden Versuchen bandelt es sich um die binoculare Ausmessung aufgeklebter Stereoskopbilder, nicht um die Ausmessung der. Wirklichkeit. Es complicirt sich die Sache insofern, als wir hier - wie wir sehen werden, nicht mit einer allgemein gültigen Scala auskommen. Da für unsere Verbältnisse in der Formel $\frac{1}{a}+\frac{1}{b}=\frac{1}{f}$ a relativ kleine Werthe hat, d. h. die Stereophotogramme aus geringer Entfernung aufgenommen werden, so fällt $b$ und $f$ nicht zusammen, $d$. h. die Bilder entstehen nicht in der Brennebene, sondern für jedes Bild haben wir eine andere Balglänge zu berücksichtigen, und dieser Balglänge entsprechend brauchen wir andere Tiefenwerthe in der Scala.

Andererseits vereinfacht sich die Messung dadurch, dass wir dann auf jedes Stereoskopbild die beiden Halbscalen, welche zu ihm der Balglänge nach passen, ohne weiteren Apparat einfach darauflegen können.

Diese einfache Methode findet freilich bald ihre Grenzen, indem sie nur auf solche Bilder anwendbar ist, die aus $1 / 3$ bis allenfalls $2 \mathrm{~m}$ Entfernung aufgenommen sind.

Bei Aufnahmen aus geringerer Entfernung, wobei wir also vergrösserte Bilder erhalten, kommen wir schon in das mikrostereophotographische Gebiet, welches noch fast ganz unbearbeitet ist. Nur bis zur achtfachen Vergrösserung habe ich experimentell die Aufnahmebedingungen festgestellt, ein objectives Messverfahren haben wir für solche Bilder noch nicht.

1) Auch erkenne ich gern an, dass in der nämlichen Mittheilung die wissenschaftliche Verwerthbarkeit der stereophotographischen Doppelaufnahmen auf eine Platte, wie ich sie in Neisser's medicinischem Atlas Lief. 46 (Beiträge zur Hirntopographie) dargethan habe, schon erörtert worden ist. Diese Arbeit war mir unbekannt geblieben. Wie mir Herr Prof. $\mathrm{Mach}^{1}$ ) liebenswürdigerweise schreibt, hätten sich Anatomen, Physiologen und praktische Optiker damals sehr ablehnend verhalten ${ }^{2}$ ). Nach seiner Erfahrung müsse dieselbe Sache oft von verschiedenen Seiten vorgebracht werden und noch dauere es 30 Jahre, bevor die Praxis davon Notiz nehme. Unter Uebersendung eines meiner Doppelstereogramme theilte ich Herrn Prof. Mach mit, dass ich durch die genannte historische Notiz seine Priorität anzuerkennen wünschte, worauf mich Herr Prof. Mach in einem freundlichen Antwortschreiben noch auf jene Mittheilung verwies.

1) Vgl. anch meine historische Notiz in den Klin. Monatsbl. f. Augenheilk. Heft Nov.

2) S. "Wozu hat der Mensch zwei Augen?"4 Populärwissensch. Vortrag. S. 85. Geh. in Graz im Jahre 1866. 
Für Aufnahmen aus grösserer Entfernung hat Pulfrich neuerdings (Zeitschr. f. Instrumentenkunde 1902, Heft 3, 5, 6 - noch nicht beendet) einen complicirten Apparat, den Stereocomparator, angegeben, in dem Stereoskopbilder z. B. des Sternhimmels der Tiefe nach ausgemessen werden können.

Meine Versuche waren vor Veröffentlichung dieser Arbeiten der Hauptsache nach abgeschlossen.

In ein mattschwarz gestrichenes Brett schlagen wir feine Nägel ein, denen der Kopf abgebrochen ist, in der Weise wie es Fig. 4 von oben zeigt: in einer Frontlinie, $2,5 \mathrm{~cm}$ von einander entfernt, zwei Nägel, von dem rechten der beiden an, in einer schrägen Linie nach links vorn fünf weitere, die sagittal gemessen, 1 bis $5 \mathrm{~cm}$ vor der Ebene der beiden oberen stehen, wenn das Brett horizontal liegt. Mit Hilfe zweier neben einander stehender Objective, deren optische Achsen horizontal und parallel gerichtet sind, bilden sich diese Nägel auf der rechten und linken Hälfte der Mattscheibe mit gewissen Differenzen $a b$, wie Fig. 4 ohne Weiteres erkennen lässt.

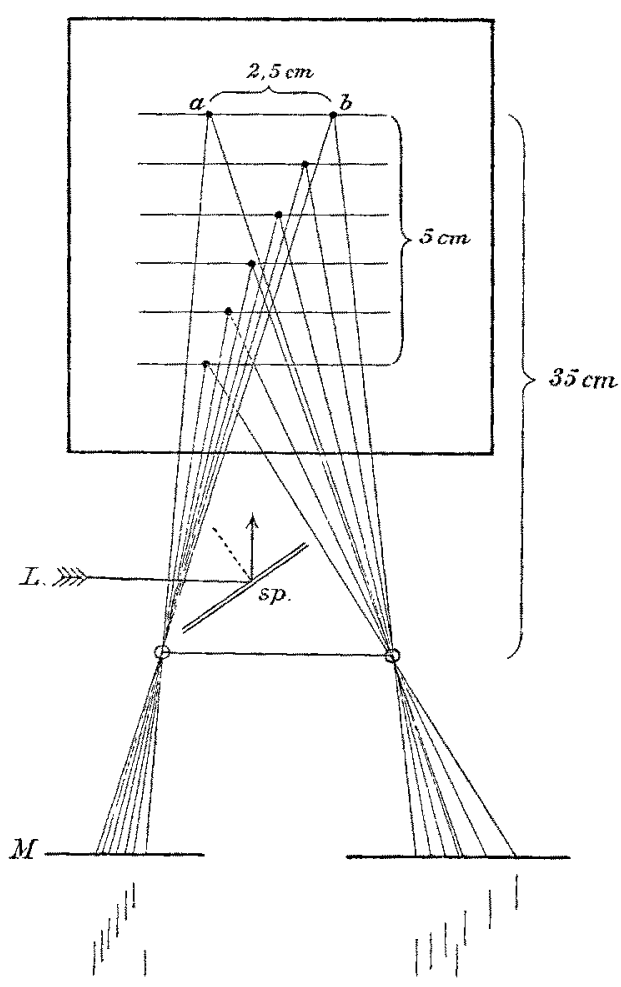

Fig. 4.

Neigen wir nun das den Objectiven zunächst liegende Ende des Brettes etwas, so erfolgt die Abbildung in der unter der Mattscheibe skizzirten Weise. Diese beiden Halbbilder, stereoskopisch verschmolzen, müssen uns den Eindruck einer von links vorn nach rechts hinten in die Tiefe verlaufenden Messscala machen, in der je zwei Marken, sagittal gemessen, $1 \mathrm{~cm}$ von einander entfernt sind.

Photographiren wir diese Markenreihe mit $17,5 \mathrm{~cm}$-Objectiven aus $35 \mathrm{~cm}$ Entfernung, so erhalten wir eine Messscala in natïrlicher Grösse, d. h. die zwei entferntesten Marken $a$ und $b$ liegen in beiden 
Halbbildern 2,5 $\mathrm{cm}$ von einander entfernt. Zur Technik sei noch Folgendes ${ }^{1}$ ) bemerkt: die Entfernung $35 \mathrm{~cm}$ ist zu messen von $a b$ zur Mitte zwischen den $64 \mathrm{~mm}$ von einander entfernten Objectiven. Vor und wenig unterhalb der Objective befindet sich ein Spiegel, der einen Lichtkegel auf die Nägel wirft. Nachdem die Balglänge genan eingestellt ist, macht man die Aufnahme bei engster Blende (wozu ich bei elektrischer Bogenlichtbestrahlung ca. 5 Secunden Exposition brauchte). Nimmt man nun irgend einen körperlichen Gegenstand aus $35 \mathrm{~cm}$ Entfernung, also in natürlicher Grösse, mit denselben $\mathrm{Ob}$ jectiven, mit $64 \mathrm{~mm}$ PD auf, so kann man auf das nach gewöhnlichen Grundsätzen angefertigte Stereoskopbild die beiden Hälften der stereoskopischen Messscala einfach auflegen. Hat man die beiden stereoskopischen Halbbilder derselben auf einer Platte neben einander aufgenommen, so muss diese natürlich zerschnitten werden. Jede Hälfte legt man nun auf das ihr zugehörige stereoskopische Halbbild und regulirt den Seitenabstand so, dass die zwei Marken $a$ und $b$ mit der „Rücken"fläche („Basis"fläche) des stereoskopischen Sammelbildes in eine frontale Ebene zu liegen kommen. Man erreicht dies am bequemsten dadurch, dass man monocular die Marke a der einen. Scala dicht neben eine markante Linie der Ruicken- oder Basisfläche des einen stereoskopischen Halbbildes legt, und dann die entsprechende Marke $a$ der anderen Scala, auch wieder monocular, dicht neben die entsprechende Linie des anderen Stereoskophalbbildes bringt. Die zwei Marken $a$ müssen dabei in einer horizontalen Linie liegen. Betrachten wir die Sache nun binocular, so wird $a$ etwa in derselben Entfernung wie die markante Linie liegen, neben die wir $a$ gelegt hatten. Durch geringe Seitenverschiebungen einer Scalenhälfte lässt sich die Einstellung genau reguliren. Hat man beide Scalenhälften durch eine horizontal laufende Schraube verbunden, so drüickt man am besten die linke Scala mit der Hand auf das Bild fest auf und regulirt den Seitenabstand der rechten durch Drehen an der Schraube mit der rechten Hand. Handelt es sich im Sammelbilde z. B. um ein Prisma, welches eine Kante dem Aufnahmeapparat zugewendet hatte, so kann man nun ohne Weiteres ablesen, mit welcher Marke der Scala diese Kante im Stereoskop in einer frontalen Ebene zu liegen scheint. Diese Marke giebt die Höhe (körperliche Tiefe) des Prismas in Centimetern an. Auf diese Weise d. h. aus $35 \mathrm{~cm}$ Entfernung habe ich nun ein Arrangement von drei verticalen Stäben, die in

1) Cfr. S. 290 Anm. 
ihrer Gruppirung ein gleichseitiges Prisma nachahmen, welches eine Kante symmetrisch dem Aufnahmeapparat zukehrt, stereophotographisch aufgenommen. Beträgt die Flächenbreite $2,5 \mathrm{~cm}$, so sollte die Höhe (körperliche Tiefe) $2,25 \mathrm{~cm}$ sein. Dies kann man mit grosser Genauigkeit mit Hilfe der Messscala finden, wenn die Pupillendistanz der (Fig. 5) Objective 64 oder $65 \mathrm{~mm}$ betrug, aber schon bei einer Verminderung der Pupillendistanz auf $55 \mathrm{~mm}$ finden wir, mit obiger Messscala gemessen, kaum $2 \mathrm{~cm}$ Höhe (Fig. 6), und erhöhen wir die Pupillendistanz auf $75 \mathrm{~mm}$, so (Fig. 7) lesen wir für das Prisma eine Höhe von $2,5 \mathrm{~cm}$ ab. Im ersteren Falle haben wir also Unter-, im zweiten Ueberplastik.

Würde man noch halbe oder viertel Centimeter in die Scala einführen, und die Linien noch feiner nehmen, so liesse sich die Feinheit der Messung ja noch weit erhöhen. Mir kommt es indess nur auf das Princip der Sache an, und ich bin überzeugt, dass es für eine solche Verfeinerung theoretisch eben keine Grenze giebt.

Es ist eine leichte Mühe, sich ähnliche Messscalen für die verschiedensten Entferriungen anzufertigen. Man photographirt stereoskopisch in der angegebenen Weise dasselbe Brett mit seinen Nägeln aus $40,50,60,75$ und $100 \mathrm{~cm}$. Von 40 oder $50 \mathrm{~cm}$ an wird man gut thun, 10 Marken, von 60 an 15 Marken anzunehmen, da es sich wohl hier schon um grössere Tiefenunterschiede handeln kann. Man kann diese Markenreihen auch vortheilhaft in Zickzackformen anordnen, wie dies im Zeiss'schen stereoskopischen Entfernungsmesser geschehen ist. Jede Scala habe ausser den sagittalen stereoskopischen Werthen auch Breitenwerthe, etwa $2 \mathrm{~cm}$ für nähere, 4 für die mittleren, $8 \mathrm{~cm}$ für entferntere Aufnahmen.

Wie weit es unbedingt nothwendig ist, dic Anfertigung der Messscalen mit denselben photographischen Objectiven vorzunehmen, mit denen die Stereoskopbilder angefertigt sind, entzieht sich vorläutig meiner Beurtheilung. Selbstverständlich muss die Balglänge (also die Brennweite der für beide $Z$ wecke benutzten Objective) genau die gleiche sein.

Man könnte gegen diese Art der Messscala einwenden: sie zeigt uns die Tiefenverhältnisse nicht richtig, sie übertreibt, weil die $O b$ jective alles, was diesseits z. B. der doppelten Brennweite liegt, zu gross zeichnen. Dies letztere ist indess ganz selbstverständlich, findet in unserem Auge in derselben Weise, nur in anderem Grade statt, und ist natürlich auch bei den verschiedenen photographischen Objectiven verschieden. Die Frage liegt auf anderem Gebiete. Sie 
heisst: Warum beurtheilen wir die uns durch die photographischen Objecte angenähert richtig übermittelten Tiefenwerthe oft so falsch?

Dass die in dem stereoskopischen Sammelbild der Scalen gegebenen Tiefenwerthe den wirklichen Entfernungsdifferenzen in der Aussenwelt durchaus entsprechen, geht aus folgendem Versuch hervor. Aus einer Entfernung von $1 \mathrm{~m}$ photographiren wir stereoskopisch eine Markenreihe, von 20 bis $25 \mathrm{~cm}$ Tiefe, mit einer gewissen Balglänge (ca. $21,5 \mathrm{~cm}$ für die von mir benutzten Objective). Die Platten bringen wir nun in dem oben geschilderten stenopäischen Stereoskop $21,5 \mathrm{~cm}$ oder, falls die photographischen Objective eine Bildverzeichnung im Sinne einer Verkleinerung der Höhen und Breitendimensionen bedingen, entsprechend näher vor die stenopäischen Löcher, und projiciren sie unter entsprechender seitlicher Annäherung in $1 \mathrm{~m}$ Entfernung an dieselbe Stelle, wo wir die Originalscala $d$. h. das Brett mit seinen Nägeln entsprechend postiren. Man kann sich leicht überzeugen, dass sich Sammelbild der Markenreihe (Scala) und Markenreihe selbst durchaus entsprechen. Dieser Versuch ist für die verschiedenen Scalen völlig übereinstimmend, ist aber auf die Scala in natïrlicher Grösse deshalb nicht anwendbar, da wir hier die Halbbilder an dieselbe Stelle setzen müssten, wo sich die Rückenfläche (Basis) der Markenreihe selbst befindet. Das Haploskop zeigt die Richtigkeit auch dieser Scala. Die in den Scalen gegebenen Werthe entsprechen also den Entfernungsdifferenzen der Wirklichkeit völlig. Die Frage ist nur die, warum taxiren wir die Tiefenwerthe der stereoskopischen Sammelbilder meist zu hoch, warum erscheinen uns die Bilder oft ïberplastisch? Diese Frage glaube ich, zum Theil wenigstens, in meiner Mittheilung über Orthostereoskopie beantwortet zu haben.

Man könnte nun wünschen, diese verschiedenen Scalen in eine einzige fortlaufende zu vereinigen, welche für alle Entfernungen zu gebrauchen wäre. Dieses ist jedoch theoretisch unmöglich und die Fehlerhaftigkeit für näher gelegene Punkte leicht zu erweisen. Photographiren wir stereoskopisch ein Prisma, welches uns eine Kante zukehrt, z. B. aus $50 \mathrm{~cm}$ Entfernung, mit der dieser Entfernung entsprechenden Balglänge, so bildet sich die beispielsweise $10 \mathrm{~cm}$ vorstehende Vorderkante in jedem Halbbild seitlich von den Bildmitten der Basisflächen unseres Prismas ab. Die Summe dieser Excentricitäten in beiden Halbbildern nennen wir die Querdisparation. Diese ist abhängig von der Balglänge. Sie ist also bei einer Balglänge, welche einer Entfernung $a=50$ entspricht (in dieser erfolgte die gedachte Aufnahme des Prismas), anders als bei einer solchen, welche 
einer Entfernung $a=40$ entspricht. Im letzteren Falle wäre sie grösser. In einer fortlaufenden Scala könnten wir nun aber jedem Punkte nur die Querdisparation geben, welche der Balglänge zukommt, die seiner eigenen Entfernung von den Objectiven entspricht. In einer solchen Scala würde also die Marke 40 eine Querdisparation haben, welche eine auf $a=40 \mathrm{~cm}$ Entfernung eingestellte Balglänge ergiebt, während sie zur Ausmessung eines aus $50 \mathrm{~cm}$ Entfernung aufgenommenen Bildes eine Querdisparation haben muss, die einer auf $50 \mathrm{~cm}$ Entfernung eingestellten Balglänge entspricht.

Denkbar und construirbar ist wohl eine sprungweise fortschreitende Scala, wie wir gleich sehen werden, doch hat diese keinen Vortheil vor mehreren Einzelscalen. Wir können stereophotographisch mit unseren $17,5 \mathrm{~cm}$-Objectiven aufnehmen aus einer Entfernung von $a=100$ bei einer Balglänge von $b=21,5$ eine $15 \mathrm{~cm}$ tiefe Markenreihe für 100 bis $85 \mathrm{~cm}$.

Diese stimmt für Bilder, die aus einer Entfernung von $100 \mathrm{~cm}$ mit $21,5 \mathrm{~cm}$ Balglänge aufgenommen sind, sie stimmt auch für solche Bilder, die ans 90 oder $85 \mathrm{~cm}$ mit derselben Balglänge aufgenommen sind, sie stimmt nicht für solche, die aus letzterer Entfernung mit der dieser Entfernung entsprechenden Balglänge photographirt sind. Andererseits dürfen wir Theilstrich 90 bis 85 in dieser Scala nicht mit der einer Entfernung von 90 oder $85 \mathrm{~cm}$ zukommenden Balglänge aufnehmen, wenn wir richtige Werthe für die Bilder haben wollen, die aus $100 \mathrm{~cm}$ aufgenommen sind ${ }^{1}$ ).

Eine zweite Scala ist aufgenommen aus

$$
a=75 \mathrm{~cm} \quad b=23 \mathrm{~cm} \quad \text { für } 75-60 \mathrm{~cm} \text { (Fig. 8). }
$$

Sie gilt für sämmtliche Bilder, die aus einer Entfernung von 65 bis $70 \mathrm{~cm}$ mit Balglänge $23 \mathrm{~cm}$ aufgenommen sind.
3. Scala
$a=60$
$b=25$
für $60-45 \mathrm{~cm}$ (Fig. 9)
4. "
$a=50$
$b=27,5$
$5 . \quad "$
$a=40$
$b=30,5$
$a=35$
$b=35$
" $50-4.0 \mathrm{~cm}$
" $40-30 \mathrm{~cm}$ (Fig. 10)
6. "
" $35-30 \mathrm{~cm}$ (Fig. 11).

Diese Scalen können wir nun alle über einander anordnen und hätten somit eine sprungweise fortschreitende Scala für Bilder, die aufgenommen sind mit den bemerkten Balglängen, nicht aber könnten wir z. B. Bịlder damit messen, die aus $45 \mathrm{~cm}$ Entfernung mit einer

1) Unter Entfernung des Gegenstandes ist hier immer die Entfernung der Rücken- oder Basisfläche, nicht etwa der vordersten Theile des körperlichen Gegenstandes von den Objectiven zu verstehen. 
dieser Entfernung entsprechenden Balglänge aufgenommen sind, denn in der Scala haben wir keine Theilwerthe, die dieser Balglänge entsprechen. $\mathrm{Zu}$ der Aufnahme aus $45 \mathrm{~cm}$ müssten wir eine Balglänge wählen, die 50 oder $40 \mathrm{~cm}$ Entfernung entspricht. Dann wäre Scala 4 oder 5 anwendbar.

Nehmen wix theoretisch an, dass wir Objecte aus den verschicdensten Entfernungen sämmtlich mit derselben Balglänge aufnehmen könnten (was für leblose Objecte mit Hilfe feinster stenopäischer Löcher möglich ist), so würden wir auf gleiche Weise eine für alle diese Aufnahmen gültige Messscala anfertigen können, indem wir mit derselben Pupillendistanz und derselben Balglänge eine lange, etwa im Zickzack angeordnete Markenreihe von 30 bis 100 oder $200 \mathrm{~cm}$ aufnehmen (Fig. 12).

\section{Resultate.}

Für die Ausmessung der Tiefenverhältnisse stereoskopischer Bilder haben wir i. O. drei Methoden kennen gelernt, die stereosko* pische Berechnung, die stereoskopische Reconstruction, die stereoskopische Messung mittels Scala.

Zur stereoskopischen Berechnung müssen wir kennen die Entfernung, aus der das Object stereophotographisch aufgenommen wurde $(a)$. Wir müssen die Brennweite der Objective $(f)$ oder die Balglänge $(b)$, ferner die Pupillendistanz der Objective kennen. Wenn wir dann mittels Massstabes die seitlichen Bildrerschiedenheiten (Querdisparation) stereoskopischer Halbbilder messen können, so ist es möglich, nach gegebener Formel die Tiefenwerthe zu berechnen. Die Fehlergrenzen sind erhebliche.

Zur stereoskopischen Reconstruction müssen wir über eine geeignete stenopäisch-stereoskopische Vorrichtung verfügen, welche uns gestattet, die stereoskopischen Halbbilder als Negative oder Diapositive in die Entfernung zu projiciren, aus der sie aufgenommen worden sind. Diese Strecke $(a)$ muss also wieder bekannt sein. Ferner muss die Pupillendistanz des Beobachters mit der der Aufnahmeobjective übereinstimmen. Auf einem sagittal verlaufenden Massstab, dessen Nullpunkt dahin gelegt ist, wo im Raume das stereoskopische Sammelbild, speciell seine Rückenfläche zu stehen kommt, verschieben wir eine Marke, bringen diese bei binocularer Betrachtung mit einem beliebigen Punkt des stereoskopischen Sammelbildes in eine frontale Ebene und lesen an dem Massstab die Tiefenwerthe ab. Die Methode giebt bei Berïcksichtigung der Eigenheiten der photographischen 
Objective sehr genaue Resultate, leidet nur unter dem Umfang des anzuwendenden Apparates.

Die stereoskopische Messung mittels Scala endlich ist leicht auszufiuhren und giebt recht genaue Resultate. Aus derselben Entfernung a, aus der ein Körper stereophotographisch aufgenommen wird, photographirt man stereoskopisch mit denselben Objectiven, derselben Pupillendistanz und gleicher Balglänge $b$. Eine Reihe sagittal, schräg oder zickzackförmig in die Tiefe und ein wenig nach oben verlaufender Marken (5 bis 15), die sagittal gemessen je $1 \mathrm{~cm}$ Abstand von einander haben. Die stereoskopischen Halbnegative dieser Scala legt man im Stereoskop jedes auf die ihm entsprechende Hälfte des zu messenden Stereoskopbildes. Bei binocularer Verschmelzung bringt man nun durch Regulirung des Seitenabstandes der Scalenbälften das Ende der Scala (den fernsten Punkt) mit der Rückenfläche des stereoskopischen Sammelbildes in eine frontale Ebene. An dem Sammelbilde der Scala kann man dann die Sagittaldistanzen einzelner Punkte des stereoskopischen Sammelbildes von dessen „Rücken"fläche oder einzelner Punkte oder Flächen von einander direct in Centimetern ablesen.

Herrn Geheimrath Uhthoff danke ich verbindlichst für sein freundliches Interesse an den vorliegenden Untersuchungen. 
Taf. IX.

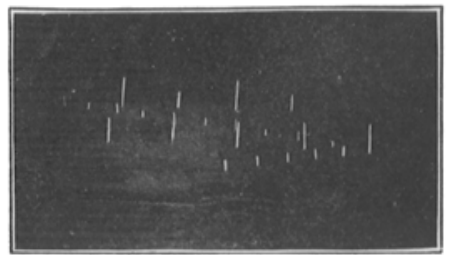

Fig. $8 L$

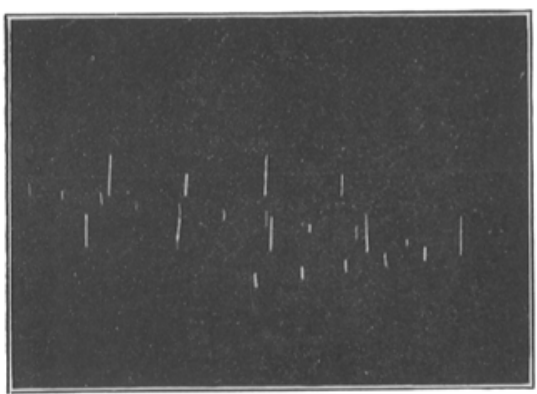

Fig. $9 x$.

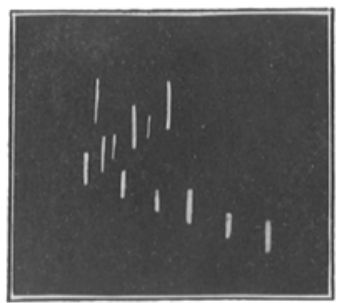

Fig. $10 \mathrm{~L}$.

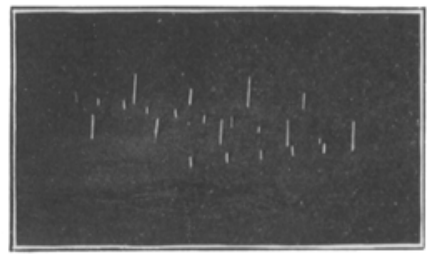

Fig. $8 R$.

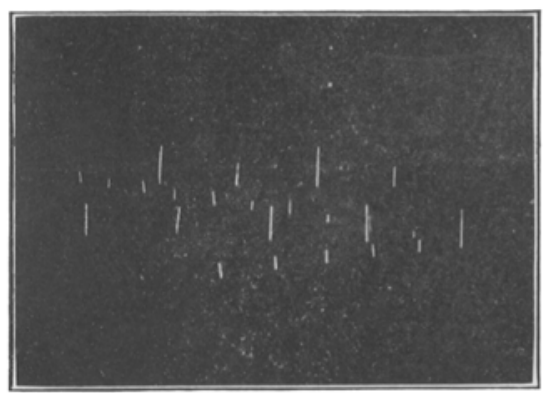

Fig. $9 R$.

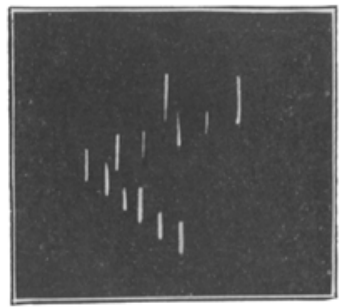

Fig. $10 R$. 


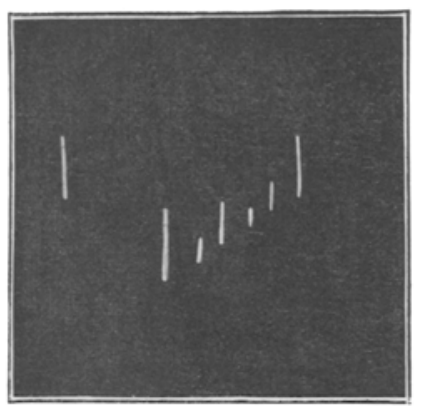

Fig. $11 \mathrm{~L}$.

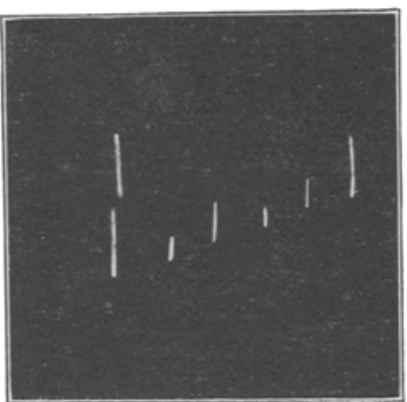

Fig. $11 R$.

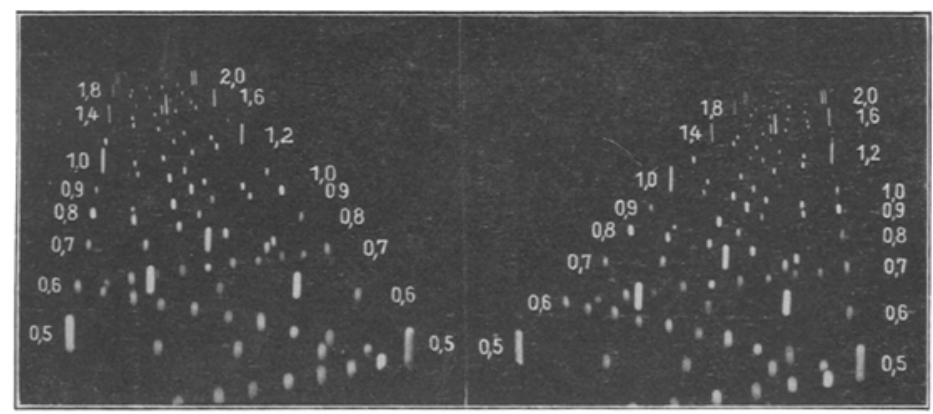

Fig. 12.

Verlag von Wilhelm Engelmann in Leipzig. 